\title{
Diabetic Retinopathy Clinical Research Network: Diabetic retinopathy and diabetic macular edema
}

\section{Red de Investigación de Retinopatía Diabética: Retinopatía diabética y edema macular diabético}

\author{
José L. Palomares-Ordóñez*, Sergio Rojas-Juárez, Jorge A. Sánchez-Ramos, Jazmín A. Salas-García, \\ Ingrid Pita-Ortíz, Angeles Y. Hernández, Carla R. Pérez-Montaño and Juan A. Ramírez-Estudillo \\ Retina and vitreous department, Fundación Hospital Nuestra Señora de la Luz, IAP, Mexico City, Mexico
}

\begin{abstract}
The diabetic retinopathy clinical research network (DRCR.net), founded by the National Eye Institute, was formed in 2002. Currently it includes over 400 ophthalmologists in 115 clinical centers in 37 states of the United States. Since then, it has conducted several studies on various points of interest about diabetic retinopathy and diabetic macular edema. To date, the DRCR.net has published about 80 articles, including clinical trials (named with alphabetic letters), observational studies and various sub analyses derived from the primary studies. The results obtained provide retina specialists with substantial evidence regarding the treatment protocols classically proposed. Also, they evaluate new treatment guidelines and information concerning other important points about the disorders studied. The large amount of information provided by the protocols and the corresponding sub analyses make understanding them a difficult task. In this work, we intend to emphasize and summarize the essential points and conclusions of each of the protocols already published by the DRCR.net, highlighting their importance in clinical practice, which will result in better decisions for the individualized treatment of diabetic patients.
\end{abstract}

Key words: Clinical trial. Analysis. Diabetic retinopathy. Diabetic macular edema.

\section{Resumen}

La Red de Investigación de Retinopatía Diabética (DRCR.net), fundada por el Instituto Nacional Ocular, se formó en 2002. Actualmente está conformada aproximadamente por 400 oftalmólogos de 115 centros clínicos de 37 estados de la Unión Americana. Desde entonces ha conducido múltiples estudios sobre diversos puntos de interés en torno a la retinopatía diabética y el edema macular diabético. A fecha de hoy, la DRCR.net tiene alrededor de 80 publicaciones, entre ensayos clínicos (nombrados con letras alfabéticas), estudios observacionales y diversos subanálisis derivados de los estudios principales. Los resultados obtenidos proveen a los especialistas en retina de evidencia con respecto a los protocolos de tratamiento clásicamente propuestos, además de estudiar nuevas pautas de tratamiento e información con respecto a otros puntos de importancia sobre las patologías estudiadas. La gran cantidad de información proporcionada por protocolo y los correspondientes subanálisis hacen de su comprensión una tarea difícil. En el presente trabajo se pretende resaltar y resumir

\section{Correspondence:}

*José L. Palomares-Ordóñez

Ezequiel Montes, 135

Col. Tabacalera, Del. Cuauhtémoc

Date of reception: 15-08-2018

Date of acceptance: 08-12-2018

E-mail: oft.jl.palomares@gmail.com 2604-1731/@ 2019 Sociedad Mexicana de Oftalmología. Published by Permanyer México SA de CV. This is an Open Access article under the CC BY-NC-ND license (http://creativecommons.org/licenses/by-nc-nd/4.0/). 
Ios puntos importantes y conclusiones de cada uno de los protocolos ya publicados por el DRCR.net, y remarcar su importancia en la práctica clínica, lo que resultará en mejores decisiones para el tratamiento individualizado de los pacientes diabéticos.

Palabras clave: Ensayo clínico. Análisis. Retinopatía diabética. Edema macular diabético.

\section{Protocol A}

The Early Treatment Diabetic Retinopathy Study (ETDRS) showed that focal or grid photocoagulation reduced the risk of moderate visual loss (MVL) caused by diabetic macular edema (DME) by $50 \%$ during three years after its application. The effectiveness of laser could not be completely explained, although it is suggested that the closure of the leak caused by the microaneurysms could be one of the most important mechanisms, at least in part. Subsequently, some clinicians observed a favorable response after the application of focal/grid laser despite not directly impacting on the microaneurysms and, likewise, there were observations of unfavorable effects after excessive applications in areas very close to the fovea. This led to the idea of generating a new treatment schedule different from that proposed by the ETDRS.

Protocol A compared two photocoagulation techniques for the treatment of DME: the modified technique proposed by the ETDRS (direct or grid laser) (group 1) versus a mild macular grid (MMG) technique (group 2). The first intends to reduce the adverse effects observed by scar expansion close to the fovea. The second is an alternative proposed by the DRCR.net, that suggests applying the spots with less power and away from the fovea, with a greater inter-spot distance. Characteristically, the spots must be applied on the apparently healthy retina, avoiding direct application on microaneurysms. Treatment techniques are detailed in Table 1.

They randomized 263 patients (323 eyes) with non-treated DME to receive one of the two photocoagulation techniques.

Visual acuity (VA) was analyzed and time-domain optical coherence tomography (TD-OCT) was performed using Stratus 3.0. Standard ETRDS fundus photographs were taken at baseline and at each visit (3.5 months, 8 months and 12 months). Fluorescein angiography (FAG) was taken at baseline and at one year. The photocoagulation technique was repeated at each visit if edema persisted.

The primary objective was to observe changes in several measures of TD-OCT at 12 months. Changes in best-corrected VA (BCVA) were considered as secondary objectives.
As a result, at 12 months it was observed that in the eyes with central macular thickness (CMT) $>250 \mu \mathrm{m}$ at baseline, CMT had a mean decrease of $88 \mu \mathrm{m}$ in group 1 versus $49 \mu \mathrm{m}$ in group $2(p=0.02)$. Macular volume decreased $0.8 \mathrm{~mm}^{3}$ and $0.4 \mathrm{~mm}^{3}$, respectively $(p=0.03)$. Mean change in BCVA was +0 letters and -2 letters, respectively $(p=0.10)$.

Lower effectiveness was achieved with the MMG technique compared to the modified ETDRS technique ${ }^{1}$. A subanalysis compared CMT with BCVA before and after treatment and found a modest correlation after treatment with laser photocoagulation. It is highlighted that VA measurement cannot be substituted even though TD-OCT is an important tool in the evaluation. Likewise, it showed that TD-OCT changes, both in the short and long term, are not predictors of VA changes ${ }^{2}$.

When correlating TD-OCT with stereo fundus photographs to evaluate retinal thickness, a weak correlation with VA was observed, as well as a moderate correlation between TD-OCT and fundus photography in evaluating CMT and a slight superiority in the reproducibility, as well as a greater sensitivity of TD-OCT to detect retinal thickness changes ${ }^{3}$.

It was also studied if the extension of DME evaluated by TD-OCT before treatment could explain the initial VA and predict the change in CMT or VA after treatment, but no answers were obtained with the subanalysis ${ }^{4}$.

When evaluating the association between FAG characteristics and VA, TD-OCT and fundus photographs, they concluded that the baseline leakage area is associated with a reduced VA and increased thickness and volume values by TD-OCT at baseline, but no associations with changes at 12 months were found ${ }^{5}$.

\section{Protocol B}

In the nineties began the research on the use of various intravitreal agents which may have a beneficial effect in different pathologies, including DME. In 2001 and 2002, the first reports of isolated cases on the use of intravitreal triamcinolone for DME treatment were published, suggesting some effectiveness. Its use began spreading according to surveys of the American Society of Retina Specialists. Since there was not a lot of evidence regarding its safety and effectiveness, it 
Table 1. Comparison of laser photocoagulation parameters for direct macular or modified grid technique proposed by the ETDRS and the mild macular grid technique by the DRCR.net.

\begin{tabular}{|c|c|c|}
\hline Spot characteristics & $\begin{array}{l}\text { Focal/grid photocoagulation (modified ETDRS } \\
\text { technique) }\end{array}$ & $\begin{array}{l}\text { Mild macular grid } \\
\text { photocoagulation (MMG-DRCR.net) }\end{array}$ \\
\hline Direct treatment & $\begin{array}{l}\text { Directly treat all leaking microaneurysms in the } \\
\text { thickened retinal area between } 500 \text { to } 3,000 \\
\text { microns from the macular center (but not } \\
\text { within } 500 \text { microns around the optic disc) }\end{array}$ & oes not apply \\
\hline $\begin{array}{l}\text { Color change of the } \\
\text { microaneurysm with direct } \\
\text { treatment }\end{array}$ & $\begin{array}{l}\text { Not required, but at least a moderate gray } \\
\text { burn, which should be evident on all } \\
\text { microaneurysms }\end{array}$ & Does not apply \\
\hline Size of spot with direct treatment & 50 microns & Does not apply \\
\hline $\begin{array}{l}\text { Duration time with direct } \\
\text { treatment }\end{array}$ & 0.05 to $0.1 \mathrm{~s}$ & Does not apply \\
\hline Grid treatment & $\begin{array}{l}\text { Applied to all areas of diffuse leakage or } \\
\text { non-perfusion within the described area of } \\
\text { treatment }\end{array}$ & $\begin{array}{l}\text { Applied to the entire described area for } \\
\text { treatment (including non-thickened retina) }\end{array}$ \\
\hline $\begin{array}{l}\text { Area considered for grid } \\
\text { treatment }\end{array}$ & $\begin{array}{l}500 \text { to } 3,000 \text { microns superior, nasal and } \\
\text { inferior from the macular center, } 500 \text { to } 3,500 \\
\text { microns temporal to the macular center. At } \\
\text { least } 500 \text { microns away from the optic disc }\end{array}$ & $\begin{array}{l}500 \text { to } 3,000 \text { microns superior, nasal and inferior } \\
\text { from the macular center, } 500 \text { to } 3,500 \text { microns } \\
\text { temporal to the macular center. At least } 500 \\
\text { microns away from the optic disc }\end{array}$ \\
\hline Spot size with grid treatment & 50 microns & 50 microns \\
\hline Duration time with grid treatment & 0.05 to $0.1 \mathrm{~s}$ & 0.05 to $0.1 \mathrm{~s}$ \\
\hline $\begin{array}{l}\text { Spot appearance with grid } \\
\text { treatment }\end{array}$ & Barely visible (light gray) & Barely visible (light gray) \\
\hline $\begin{array}{l}\text { Inter-spot distance with grid } \\
\text { treatment }\end{array}$ & Separation of two burn diameters & $\begin{array}{l}\text { Total of } 200 \text { to } 300 \text { burns ( } 2 \text { to } 3 \text { burn diameters } \\
\text { of separation) }\end{array}$ \\
\hline $\begin{array}{l}\text { Wavelength of focal or grid } \\
\text { treatment }\end{array}$ & Green to yellow wavelengths & Green to yellow wavelengths \\
\hline
\end{tabular}

was considered important to conduct a randomized clinical trial.

The study consisted in comparing triamcinolone with the then gold-standard proposed by the ETDRS for DME, focal or grid laser photocoagulation.

Patients with DME involving the fovea $(n=840)$ were randomized into 3 groups: 1) $1 \mathrm{mg}$ of intravitreal triamcinolone, 2) $4 \mathrm{mg}$ of intravitreal triamcinolone or c) fo$\mathrm{cal} / \mathrm{grid}$ photocoagulation. The patients were treated at the beginning of the study and if necessary, retreated at each visit (with a 4-month interval). The triamcinolone groups could receive rescue laser in case of fulfilling failure criteria.

Those patients with visual impairment of 15 letters or more on two consecutive visits separated by 4 months (MVL) could receive an alternative treatment at investigators discretion (patients in the laser group could receive triamcinolone and those in the triamcinolone groups could receive laser). Results at 4 months showed a greater visual improvement in the $4 \mathrm{mg}$ triamcinolone group compared to the other two; however, at one year of follow-up, there was no significant difference between the three groups.

At 2 and 3 years of follow-up ( $n=306$ ), there was a greater VA improvement in the laser group compared to the two triamcinolone groups. This difference was not considered attributable to the development of cataracts since the results were consistent when analyzing pseudophakic patients. In addition, CMT decrease was similar in the three groups. This study showed that focal/grid laser has greater long-term benefits compared to intravitreal triamcinolone in patients with DME, in addition to having less adverse effects (increased intraocular pressure and cataract progression) ${ }^{6,7}$.

\section{Protocol C}

Some studies showed variations in retinal thickness due to OCT throughout the day in some patients with DME: it was higher in the morning and lower in the later hours of the day. Because OCT has become the standard tool for the evaluation of DME, it was considered 
important to study its diurnal variation for a more accurate interpretation.

In this study $(n=156)$, the retinal thickness was measured using TD-OCT at the following time points: 8 , $10,12,14$ and $16 \mathrm{~h}$. The average central subfield thickness (CST) at $8 \mathrm{~h}$ was $360 \mu \mathrm{m}$, with a decrease of $6 \%$ $(13 \mu \mathrm{m})$ at $16 \mathrm{~h}$. In 5 eyes (3\%) there was a decrease of more than $25 \%$ of the retinal thickness. The retinal thickness change was significantly greater in eyes with the highest retinal thickness at $8 \mathrm{~h}$. In $10 \%$ of the eyes there was a gain of more than 10 ETDRS letters and a loss of 10 letters in $6 \%$ of the eyes examined. According to the results of this study, it is considered that, although there may be a progressive decrease in retinal thickness throughout the day in patients with DME, these changes are small, occasional, and of little clinical relevance, so they should not be considered for scheduling measurements in clinical trials ${ }^{8}$.

\section{Protocol D}

The vitreous humor has been implicated, at least in part, as a cause of macular edema (ME) by various physiological and mechanical mechanisms. Likewise, it was observed that the separation between the vitreous and the retinal surface resulted in a subsequent reduction of the retinal thickness in patients with DME, either by surgery or by spontaneous detachment. Therefore, the DRCR.net performed a prospective observational study $(n=87)$ to evaluate the visual and anatomical results (CMT by TD-OCT) after vitrectomy without concomitant cataract surgery in patients with DME associated with vitreomacular traction (VMT). In addition to vitrectomy, an epiretinal membrane (ERM) peeling was performed in $61 \%$ of the cases, internal limiting membrane (ILM) peeling in 54\%, panretinal photocoagulation (PRP) in $40 \%$, and triamcinolone injection at the end of the procedure in $64 \%$. Mean baseline BCVA was 20/100 and baseline CMT was $491 \mu \mathrm{m}$. At 6 months, there was a mean CMT reduction of $160 \mu \mathrm{m}$ and $68 \%$ of the patients had a reduction of at least $50 \%$ in thickness. Thirty-eight percent of the patients showed a gain of $\geq 10$ letters, although $22 \%$ presented a loss of $\geq 10$ letters. After 6 months no significant changes were observed.

These results suggest that it is beneficial to consider vitrectomy for the management of DME in eyes with MVL associated with VMT since most of the eyes showed a reduction in CMT and between 28 and $49 \%$ of the eyes showed significant visual improvement. It is worth mentioning that between 13 and $31 \%$ of the eyes showed significant visual worsening ${ }^{9}$.

When analyzing the associated factors in those eyes that showed the greatest VA gain, it was observed that VA gain occurred in eyes with the worse initial BCVA and in those with an ERM peeling, so it is suggested that ERM peeling could be beneficial. The presence of vitreoretinal interface abnormalities is associated with a greater reduction in CMT but without significant differences in the final $B C V A^{10}$.

\section{Protocol E}

Injections of peribulbar corticosteroids were used for the treatment of ME secondary to uveitis, with favorable results, so their efficacy was suggested in DME. It was thought that peribulbar administration had fewer adverse effects than the intravitreal route, which would justify their use specifically in patients with DME and good vision, in whom the risks of intravitreal injections did not justify treatment application. Therefore, a pilot study $(n=129)$ was conducted to evaluate the effects of these drugs at the anterior and posterior sub tenon level, with or without adjuvant photocoagulation, in patients with DME and good VA ( $\geq 20 / 40$ ). Patients were randomized in 5 groups: 1) focal photocoagulation, 2) anterior subtenon injection of $20 \mathrm{mg}$ triamcinolone, 3) anterior subtenon injection of $20 \mathrm{mg}$ triamcinolone followed by focal photocoagulation after 4 weeks, 4) posterior subtenon injection of $40 \mathrm{mg}$ triamcinolone and 5) posterior injection of $40 \mathrm{mg}$ triamcinolone followed by focal photocoagulation after 4 weeks. The results at 34 weeks of follow-up did not show a significant difference in retinal thickness or VA between the 5 groups, and adverse effects attributable to the injections were reported, such as increased intraocular pressure and palpebral ptosis. In conclusion, peribulbar triamcinolone injections did not show any significant benefit in patients with DME and good VA ${ }^{11}$.

\section{Protocol F}

The DRS and ETDRS studies showed that PRP reduces the risk of severe visual loss $(<5 / 200$ in 2 consecutive visits with a 4-month interval), so it became the gold-standard for proliferative diabetic retinopathy (PDR). But this treatment is not innocuous since several complications have been reported (e.g., onset or worsening of ME) and there is not a standardized number of PRP sessions. 
The objective was to compare the effects of PRP on DME applied in a single session (group 1) versus 4 sessions (group 2) in patients with early PDR or severe non-proliferative diabetic retinopathy (NPDR) with relatively good VA (>20/32) and CMT $<300 \mu \mathrm{m}$ by TD-OCT.

In $18 \%$, preexisting ME was determined. During follow-up, retinal thickness tended to increase slightly compared to baseline levels, but CMT was slightly higher in group 1 on the day $3(+9$ versus $+5 ; p=0.01)$ and week $4(+13$ versus $+5 ; p=0.003)$, and at week 34 , the difference was reversed, and the thickness was slightly increased in group $2(+14$ versus $+22 ; p=0.06)$. VA results were similar to CMT results. At 34 weeks, VA was slightly worse in group 2 (0 versus -2 letters; $p=0.006$ ) compared to baseline. Despite the changes reported by TD-OCT, only one eye in each group required focal/grid laser.

As in other studies reported, the results did not show significant differences in the effect of treatment on VA. No adverse effects were identified in any group. In conclusion, PRP can be safely applied in a single session; however, this study does not provide enough evidence to show superiority when compared to several sessions ${ }^{12}$.

\section{Protocol G}

$M E$ is the main cause of MVL in diabetic patients. The diagnosis is clinical and is complemented by macular OCT. The indication to treat clinically significant DME is widely accepted, but the value of identifying subclinical DME is unknown. Subclinical DME with central involvement was defined as macular thickening by TD-OCT from $\geq 225$ to $\leq 299 \mu \mathrm{m}$, but not observed on clinical examination.

In a first study carried out in 2008, the objective was to evaluate CMT by TD-OCT in diabetic patients without diabetic retinopathy (DR) or with mild NPDR (only microaneurysms), without central macular thickening $(<225 \mu \mathrm{m})$ and with a VA better than 20/32 $(n=97)$ and compare it with reference values in a population without retinal disease. The average CMT was $201 \pm 22 \mu \mathrm{m}$ and was slightly higher in men. No other factors, apart from gender, were found associated with an increased central macular thickness. Concluding that in this group of patients, CMT does not differ from the reference values of a population without diabetes and that gender differences must be considered for thickness evaluation ${ }^{13}$.

An observational study conducted in 2012 ( $n=43)$ determined the progression rate from subclinical DME to clinically significant DME (CMT increase of $50 \mu \mathrm{m}$ or CMT $\geq 300 \mu \mathrm{m}$ ) or DME requiring treatment. At 1 year, 16/43 eyes met the criteria for clinically significant ME. The probability of having a CMT increase at 1 year was $27 \%$ and at 2 years was $38 \%$. It was demonstrated that subclinical ME is uncommon (4.8\%), but that it has a higher percentage (1/4 to $1 / 2$ to 2 years) of progression to clinically significant ME than that reported by the ETDRS ( $25 \%$ at 3 years). Monitoring of these patients is suggested because of their high risk of progression ${ }^{14}$.

\section{Protocol H}

Bevacizumab is a humanized monoclonal antibody that inhibits all isoforms of vascular endothelial growth factor A (VEGF-A). It has been widely used off-label for the treatment of DME.

This was the first phase 2 study to evaluate the effect of bevacizumab in DME. One hundred and twenty-one patients were randomized to 5 groups: 1) baseline focal laser, 2) $1.25 \mathrm{mg}$ bevacizumab at baseline and 6 wee$\mathrm{ks}$, 3) $2.5 \mathrm{mg}$ bevacizumab baseline and at 6 weeks, 4) $1.25 \mathrm{mg}$ bevacizumab baseline and sham reinjection (placebo) at 6 weeks, or 5) $1.25 \mathrm{mg}$ bevacizumab baseline and at 6 weeks plus focal laser at 3 weeks. The first phase (12 weeks) evaluated the efficacy and the second phase evaluated safety up to 70 weeks. The primary objective was CMT by TD-OCT and BCVA at 24 weeks. Groups 2 and 3 had the greatest reduction in CMT at 3 weeks and improvement of 1 line of BCVA at 12 weeks, compared with the laser group alone, with no differences observed between groups 2 and 3 . The combination of laser with bevacizumab showed no short-term benefits, but no adverse effects either. Regarding adverse effects, there was a case of endophthalmitis, two cases of acute myocardial infarction and one event of congestive heart failure. The conclusions were that bevacizumab reduces CMT more quickly while laser reduces it more slowly, but after 3 weeks the effect is similar. There is no short-term difference between both doses in CMT reduction, and the effect of bevacizumab has an initial peak that is stationary, or even decreases, at 3 and 6 weeks, suggesting that 6 weeks is a long time for a second dose. This study was the basis for the subsequent phase 3 studies conducted with bevacizumab ${ }^{15}$.

\section{Protocol I}

The benefits of focal or grid laser for the treatment of DME demonstrated by the ETDRS in 1985 were verified 
by the DRCR.net (protocol B), remaining as the gold standard versus triamcinolone, after excluding the group of phakic patients who developed lens opacity. However, in those patients with a VA $\leq 20 / 40$, approximately $20 \%$ of those treated with focal or grid laser presented a loss of $\geq 2$ lines. This explains the need for new therapies that could have a greater benefit, such as monoclonal antibodies, either in monotherapy or as adjuvant therapy to photocoagulation.

Protocol I is a multicentric clinical trial in which 854 study eyes were randomized from 691 participants with diagnosis of DME with central involvement. The main objective of the study was to evaluate the effect of ranibizumab or triamcinolone in combination with laser, compared with laser monotherapy, for the treatment of $\mathrm{DME}^{16}$.

Subjects were randomized in 4 groups: 1) simulated intravitreal injection plus focal or grid early laser (within 7-10 days) ( $n=293)$, 2) $0.5 \mathrm{mg}$ ranibizumab plus early laser $(n=187)$, 3) $0.5 \mathrm{mg}$ ranibizumab plus delayed laser (>24 weeks) $(n=188)$, or 4$) 4 \mathrm{mg}$ triamcinolone plus early laser $(n=186)$.

The treatment protocol for laser photocoagulation was administered according to the modified technique of the original protocol proposed by the ETDRS, previously described and used in previous protocols of DRCR.net; while the treatment protocol with the intravitreal agents was carried out in the following way: from the beginning of the treatment until week 16, injections were applied every 4 weeks regardless of VA or macular thickness; from weeks 16 to 20 a monthly application was maintained, except when "success criteria" were met (VA> 20/20, CMT <250 $\mu \mathrm{m}$ ); from week 24 to 48 , patients with "success criteria" were categorized and treatment was delayed, those with improvement (VA gain of $>5$ letters or improvement of CMT $<10 \%$ ) continued monthly injections. Those without improvement were assigned to treatment at the discretion of the investigator and, finaIly, those with failure (VA loss of 10 letters compared to baseline, CMT > 250 $\mu \mathrm{m}$ and without improvement 13 weeks after completing the laser treatment) were assigned an "alternative" treatment at the discretion of the investigator. From week 48 , visits were scheduled every 4 months, depending on the case. If treatment had been delayed in previous visits, the follow-up was extended to twice the time of the previous visit, with a maximum interval of up to 16 weeks.

The results from the first year showed the superiority of ranibizumab, either with an early laser $(+9 \pm 11)$ or with a delayed laser $(+9 \pm 12)$, for improving VA compared to a combined therapy of triamcinolone plus early laser $(+4 \pm 13)$ or laser monotherapy $(+3 \pm 13)$. The reduction in mean CMT by TD-OCT was similar for ranibizumab or triamcinolone. Likewise, in pseudophakic patients $(n=273$ ), the visual improvement was comparable for both therapies ${ }^{16,17}$.

After 3 years of follow-up, superiority was maintained in the ranibizumab groups with an early or delayed laser, and they presented a difference in mean VA of +2.9 letters in the delayed laser group compared to the early laser group. Likewise, the delayed laser group showed a greater percentage of patients with a gain of $>10$ letters $(56 \%)$ compared with the early laser group $(42 \%)^{18}$.

The results at 5 years of follow-up showed proportions similar to the initial differences: ranibizumab with early $(+7.2$ letters) or delayed ( +9.8 letters) laser showed a mean difference of -2.6 letters $(p=0.009)$. The subjects of the ranibizumab plus early or delayed laser group required an average of 8 to 9 injections during the 1st year, 3 to 4 during the 2nd year and 1 to 2 during the 3rd year, respectively. For years 4 and 5 , the mean number of injections was 0 for both groups.

Likewise, those eyes assigned to the ranibizumab treatment groups had a lower rate of progression from severe NPDR to PDR (8 versus $42 \%$ ) even when the injection was not applied monthly after week 12 , compared to those assigned to the early laser monotherapy group. Therefore, it was also shown a beneficial effect of ranibizumab for the treatment of neovascularization, which suggests that indefinite monthly injections are not necessary to maintain these beneficial effects ${ }^{19}$.

\section{Protocol J}

The worsening of pre-existing DME after PRP is a recognized adverse effect, although infrequent. VEGF has a role in the development and exacerbation of DME, so steroids and anti-VEGF drugs may reduce the exacerbation of pre-existing DME in patients with PDR and severe NPDR.

For this study, 345 eyes were randomized with a VA $\geq 20 / 320$ and DME treated with focal/grid laser and with DR under treatment with PRP, into 3 groups: 1) baseline sham injection at 4 weeks, 2) $0.5 \mathrm{mg}$ ranibizumab at baseline and 4 weeks, and 3) baseline triamcinolone and sham injection at 4 weeks, to evaluate the shortterm effect on DME. At 14 weeks, the mean change in VA was $-4 \pm 14$ letters, $+1 \pm 11$ letters and $+2 \pm 11$ letters in each group, respectively, with a greater proportion of eyes with improvement ( $>10$ letters) and a lower proportion with worsening (> 10 letters) in groups 2 and 3 , respectively. The changes in retinal thickness 
paralleled those of VA. Subsequent evaluations at 34 and 56 weeks only aimed to assess long-term safety, although it was observed that differences in VA and CMT did not persist until 56 weeks. This study concluded that the addition of triamcinolone or ranibizumab to focal laser plus PRP, is associated with an increased VA and a greater CMT decrease at 14 weeks but it was not able to determine the long-term benefit of this treatment ${ }^{20}$.

\section{Protocol K}

The criteria for retreating with focal/grid laser are an important aspect, and to some extent, there was limited information regarding the results obtained after a single laser session. The ETDRS and the DRCR.net established the criteria for retreatment (persistence of clinically significant ME, identification of one or more treatable lesions that could be the cause of visual loss) at 4-month intervals.

The objective of this study was to determine if CMT reduction associated with a single session of focal or grid laser would persist even after delaying treatment in patients with central involvement of DME after week 16. Treatment consisted in focal or grid laser photocoagulation in 122 eyes with clinically significant ME according to the modified ETDRS technique. From week 16 , patients were followed-up every 8 weeks and laser treatment was delayed if they showed a gain of $>5$ ETDRS letters or a CMT decrease $>10 \%$ compared to baseline.

At week 16, 47\% showed a VA improvement. Of these, $48 \%$ ( 26 eyes) had a CMT $\geq 250 \mu \mathrm{m}$ and were monitored until week 32 . Only 11/26 eyes showed a CMT decrease of $>10 \%$ between week 16 to 32 , with no need for retreatment.

This study demonstrated that 23 to $63 \%$ of the eyes that showed improvement but not a complete resolution of DME at 16 weeks after laser application, will continue to improve without needing retreatment until 32 weeks. This is useful evidence to assess a retreatment schedule ${ }^{21}$.

\section{Protocol L}

The ETDRS, in addition to providing solid evidence concerning DR and DME, established a standardized method for the assessment of manual optical refraction and thus BCVA (important research objectives). However, the methodology presents certain difficulties, among which can be highlighted the need for trained personnel, long time for the refraction and significant costs. An alternative to this, facilitated by technological advancement, could be autorefraction.

This study included prospectively 878 eyes of 456 participants from 26 centers. The main objective was to compare the VA obtained after performing automated refraction with different autorefractors versus the manual refraction (MR) performed according to the DRCR.net protocol in patients with visual loss secondary to DME with a wide range of VA (Snellen equivalent better than 20/400). The VA score was determined with the electronic VA test of the ETDRS. All the participants underwent the VA test on three occasions: on measurement with autorefraction and two additional measurements using manual refraction.

The spherical equivalent obtained was similar with both techniques, with a mean difference of 0.00 and a range of -1.75 to 1.13 diopters. But average VA was slightly better with manual refraction. The variability obtained between both techniques (automated refraction versus manual refraction) was considerably higher than between the two manual measurements $(p<0.001)$ and was highly dependent on the autorefractor model. The smallest variability for automated refraction was obtained with the Topcon 8000 series.

The autorefractors used in this study were not able to replace manual refraction, mainly for clinical trials due to the high variability ${ }^{22}$.

\section{Protocol M}

Although it has been established through various studies that intensive glycemic control can reduce the frequency and progression of microvascular complications in individuals with diabetes mellitus (DM), it is clearly difficult to achieve, especially in the long term. Some authors point out obstacles that prevent optimal glycemic control, highlighting the lack of awareness about the potential damage to target organs, cultural aspects, as well as financial limitations, poor access to health services, depression, lack of perseverance and others ${ }^{23-26}$.

The DRCR.net carried out a study in 42 study centers. The objective was to determine whether the combination of personalized risk assessments and diabetes education in an ophthalmology clinic could improve glycemic control in people with diabetes, assessed by glycosylated hemoglobin ( $\mathrm{HbA1c}$ ) with a primary cut-off point at 1 year, extended to 2 years.

Diabetic patients were randomized to two groups: one that received the intervention and the other with control patients treated with standard care. Likewise, 
each group was subdivided into those with more frequent than annual follow-up and those with annual follow-up. The patients in the intervention group received evaluation and personalized education, which consisted of: a) cut-off point for HbA1c, b) information on blood pressure and the severity of diabetic retinopathy (DR), c) personal risk for DR progression based on $\mathrm{HbA1c}$ and type of diabetes, d) a personalized report of the risk of kidney damage and retinopathy based on $\mathrm{HbA1c}, \mathrm{e})$ a chart with previous and recent reports of $\mathrm{HbA1c}, \mathrm{f})$ instructions to indicate the individual risks encountered and g) supplementary material for the management of diabetes. The patients in the control group were required to complete a minimum an annual visit without receiving more education than usual in the ophthalmological visit.

The mean $\mathrm{HbA1c}$ report in patients with more frequent than annual follow-up was $8.3 \%$ in the control group $(n=502)$ and $8.6 \%$ in the intervention group $(n=488)$, with an annual change of $-0.1 \%$ and $-0.3 \%$, respectively, while patients with annual follow-up had a mean $\mathrm{HbA1c}$ of $8.3 \%$ in the control group $(n=368)$ and $8.4 \%$ in the intervention group $(n=388)$, with a change of $0.0 \%$ and $-0.1 \%$, respectively. In conclusion, this study corroborated the difficulty of making a change in the personal behavior of patients, even with adequate education about the pathology and its possible complications, also without presenting differences between annual or more frequent follow-up ${ }^{27}$.

\section{Protocol N}

Vitreous hemorrhage secondary to PDR is considered one of the main indications for vitrectomy in the USA, carried out when the hemorrhage prevents the adequate application of PRP. Despite technological advances in retinal surgery, complications cannot be ignored. Some series of uncontrolled cases suggest that intravitreal antiangiogenics clear vitreous hemorrhage in the short-term so PRP can be applied without requiring vitrectomy.

This study evaluated the effect of intravitreal ranibizumab versus intravitreal saline to determine if ranibizumab increases the clearance rate of vitreous hemorrhage and thus avoids the need for vitrectomy. It was injected at baseline and at 4 and 8 weeks. At 16 weeks, which was the first cut-off point, the probability of vitrectomy was of $12 \%$ for the ranibizumab group and $17 \%$ for the saline group $(p=0.37)$. The main cause of vitrectomy between weeks 8 and 12 was persistent vitreous hemorrhage and, in a low proportion, progression to tractional retinal detachment (TRD). There was also a marked improvement in VA, a greater cumulative possibility of PRP completion and a lower recurrence rate of vitreous hemorrhage, suggesting a short-term biological effect of ranibizumab ${ }^{28}$.

Subsequently, in the 1-year analysis, the difference in VA did not persist and there were no relevant clinical differences between both groups, with a slightly greater possibility of PRP completion in the group treated with ranibizumab ${ }^{29}$.

\section{Protocol 0}

It is important for clinical practice, as well as in the field of research, to know exactly what is the normal CMT in the eyes of individuals with DM but without DME and to differentiate it from those with DME by OCT.

In a previous study, it was observed that patients with DM without DR or with mild DR, as well as those with DR without DME, have thickness values similar to patients without DM and with apparently normal retinas. All this was determined by TD-OCT (OCT-3, Stratus).

They performed a first study that evaluated the macular thickness of people diagnosed with DM, without DR or with minimal DR using spectral-domain OCT (SD-OCT, Spectralis, Heidelberg Engineering, Inc.).

Comparative measurements between the Spectralis and Stratus of the 9 ETDRS subfields were made, as well as a comparison with a reference database previously reported by the DRCR.net.

CMT mean $(n=122)$ was $270 \pm 24 \mu \mathrm{m}$, with the central point in the range of $227 \pm 25 \mu \mathrm{m}$, and the mean volume was $8.4 \pm 0.4 \mathrm{~mm}^{3}$. Characteristically, men presented greater thicknesses and greater volumes. In contrast, the Stratus reported a mean CMT of $199 \pm 24$ $\mu \mathrm{m}(71 \mu \mathrm{m}$ less than Spectralis) and an average volume of $6.7 \mathrm{~mm}^{3} \pm 0.4 \mathrm{~mm}^{3}\left(1.7 \mathrm{~mm}^{3}\right.$ smaller). The results were comparable with data obtained from healthy individuals without $D M$.

This study reported the mean thickness found in each of the 9 ETDRS macular field areas. Thicknesses $\geq 320 \mu \mathrm{m}$ in men and $\geq 305 \mu \mathrm{m}$ in women are proposed as gender-specific thicknesses to determine ME with central involvement of diabetic origin ${ }^{30}$.

The reproducibility of retinal thickness by TD-OCT and SD-OCT was evaluated, and equations were made to convert the measurements obtained by Stratus and determine if they are equivalent to SD. It was determined that there is a better reproducibility of the measurements with Spectralis compared to Cirrus or Stratus ${ }^{31}$. And when evaluating the reproducibility between CMT 
obtained by Stratus or by Optovue RTVue, it was concluded that the variability of RTVue is similar to the one of Stratus or Cirrus ${ }^{32}$.

The analysis provided by the network is useful for evaluating and comparing the results between trials and for the development of specific cut-off points of the different SD-OCTs available for subsequent clinical trials.

\section{Protocol P}

Even when cataract surgery is performed in diabetic patients routinely, there is limited data on VA and the behavior of CMT after said surgery in patients with DME.

This was a pilot, observational study with the main objective of assessing VA and the behavior of ME after cataract surgery in the eyes of patients previously diagnosed with DME with central involvement. They included 63 patients diagnosed with DME in at least 1 eye (CMT > $250 \mu \mathrm{m}$ by TD-OCT, $>310 \mu \mathrm{m}$ by SD-OCT). Thirty-five percent $(n=21)$ of the patients did not receive treatment for macular edema during the study. Forty-three percent $(n=26)$ received treatment before surgery (focal/grid laser, intravitreal triamcinolone within 4 months prior to surgery, intravitreal antiangiogenics within 2 months prior to surgery). Seven percent $(n=4)$ received intraoperative treatment and $42 \%$ $(n=25)$ received postoperative treatment. Of all patients who received treatment, $69 \%(n=27)$ received intravitreal antiangiogenics.

At the end of the follow-up, at week 16, a change in the mean VA of +12 letters was observed $(95 \% \mathrm{Cl}:+8$ to +16 ), a gain of at least 4 lines was observed in $32 \%$ (95\% Cl: $20-45 \%)$ of the eyes, with a VA worsening of at least 2 lines in 10\% (95\% Cl: 4-21\%). Secondarily, a change in the mean CMT by OCT of $-11 \mu \mathrm{m}(95 \% \mathrm{Cl}$ : -51 to $28 \mu \mathrm{m}$ ) at 16 weeks was observed.

This study has the limitation of a small sample size associated with a heterogeneous treatment for DME; however, with the results obtained, it is estimated that at least half of the operated eyes that had previous diagnosis of DME may not show visual improvement. Due to the low level of evidence, it is not possible to obtain definitive conclusions in this study ${ }^{33}$.

\section{Protocol Q}

It is widely accepted that DM increases the incidence of cataracts and may increase the risk of poor vision after surgery. On the other hand, cataract surgery has been reported as a risk factor for the incidence of DME or for the progression of DR.
The objective of this study was to determine the incidence of ME with central involvement after cataract surgery in patients with DR without pre-existing central macular thickening. This study enrolled 293 patients into 4 groups according to OCT: 1) without DME, defined as a central macular thickness less than the normal mean in all subfields; 2) "possible" DME in the central field, defined as a thickness between the mean and +2 standard deviations from the normal; 3) non-central "possible" DME, defined as a thickness of at least 1 non-central field between the mean and +2 standard deviations, and 4) "definitive" non-central DME, defined as at least one non-central field of at least +2 standard deviations.

The results were evaluated 16 weeks after surgery. It started with 329 participants; however, after adjusting for exclusion criteria and for the follow-up at 16 weeks, data from 261 eyes were analyzed. No eyes with previous DME developed EM with central involvement. Ten of the 97 (10\%, 95\% Cl: 5-18\%) eyes without central involvement prior to surgery developed central DME, 18 of the 147 (12\%, 95\% Cl: 7-19\%) eyes with "possible" central involvement developed central DME. The rate of development of DME with central involvement 16 weeks after cataract surgery differed according to the history of previous treatment for DME $(p<0.001)$. Of the patients who had been previously treated for DME, $21 \%$ (95\% Cl: $14-30 \%)$ developed central ME, while $4 \%$ (95\% Cl: $29 \%)$ of those who did not have a history of prior treatment developed central ME. As for DR, $82 \%$ did not present changes, while $10 \%$ progressed. It was also found that those eyes with better VA and less severe $D R$ at the beginning of the study had a lower incidence of central DME.

In conclusion, it was demonstrated that there is a higher possibility of developing DME with central involvement after cataract surgery in patients with non-central ME at baseline, as well as with history of previously treated DME with central involvement ${ }^{34}$.

\section{Protocol R}

DME is the most common cause of MVL in patients with DR. The increase of several cytokines and other proinflammatory factors plays a role in DME explaining, at least in part, the pathophysiology of the disease. Non-steroidal anti-inflammatory drugs have been used for postoperative inflammation. Topical nepafenac is frequently used for the treatment of postoperative cystoid macular edema. 
The main objective of this study was to evaluate the role of topical $0.1 \%$ nepafenac in eyes with non-central DME, defined as thickening within $3,000 \mu \mathrm{m}$ from the center, without involving the fovea.

Patients were randomized into 2 groups $(n=125)$. Group $1(n=61)$ received nepafenac 3 times a day. Group $2(n=64)$ received placebo. The initial follow-up was 12 months and treatment for DME was not allowed unless central involvement was observed.

The initial mean volume by TD-OCT was $7.8 \mathrm{~mm}^{3}$ and mean central thickness was $223 \mu \mathrm{m}$. The change at 12 months was $-0.03 \mathrm{~mm}^{3}$ in group 1 and $-0.02 \mathrm{~mm}^{3}$ in group 2, without differences when analyzed after adjusting for state of the lens and HbA1c. In group 1, 7 eyes $(11 \%)$ developed central DME, while in group 2 nine eyes (14\%) developed it. Of which 5 eyes in group 1 and 3 eyes in group 2 required treatment.

The average change in VA was 0.09 letters and -0.15 letters, respectively, with a difference of 0.2 letters between the groups $(p=0.82)$. Among the side effects was a case of corneal lysis in one eye of the group treated with nepafenac, with a history of severe dry eye.

In conclusion, no benefit was found with the topical use of nepafenac for 12 months in terms of retinal thickness by OCT or VA. Nepafenac appears to be well tolerated, without showing differences in adverse effects compared to the control group ${ }^{35}$.

\section{Protocol S}

PRP has been the standard treatment for PDR since the reports by the DRS and the ETDRS. Currently, with the advent of intravitreal antiangiogenic therapy, new alternative treatments have been proposed, based on the antiangiogenic effect of these drugs.

Protocol $\mathrm{S}$ evaluated the noninferiority of $0.5 \mathrm{mg}$ ranibizumab (Lucentis, Genentech) versus PRP for the treatment of PDR with or without DME in patients without previous PRP. Participants were randomized $(n=305$, 294 eyes) into 2 groups. Group 1 received PRP, completed in 1 to 3 visits, and group 2 received ranibizumab at baseline and every 4 weeks if they met the retreatment criteria, and could receive rescue laser if needed. In case of DME, group 1 could receive antiangiogenic treatment. In case of vitreous hemorrhage or tractional retinal detachment, vitrectomy was indicated.

The main result was a BCVA gain of +2.8 letters in group 1 compared to +0.2 in group $2(p<0.001)$ after 2 years of follow-up. Also, patients in group 1 showed increased visual field loss. Vitrectomy was performed in $15 \%$ of patients in group 1 and in $4 \%$ in group 2 .
More patients in group 1 developed DME (28 versus $9 \%$ ) and $53 \%$ of patients in group 1 also received ranibizumab and only $6 \%$ of group 2 received PRP.

At 2 years there was no statistical difference in terms of activity or regression of neovascularization between both groups. There were no differences in the rates of retinal detachment, neovascular glaucoma or vitreous hemorrhage between both groups. The vitrectomy rate was significantly higher in the PRP group (15\%) than in the ranibizumab group $(4 \%)^{36}$.

In a cost-benefit analysis in terms of monotherapy with ranibizumab or PRP for PDR, it was observed that it was more appropriate to initiate monotherapy with PRP for patients with PDR but without associated DME, and ranibizumab for those with DME at the time of diagnosis ${ }^{37}$.

The 5-year results $(n=184)$ show that the average number of injections in group 1 was 7.9 and in group $2,19.2$. The mean change in VA was +10.5 versus +14.3 letters $(p=0.68)$, and the mean final BCVA in both groups was 20/25, which was consistent with the data at 2 years. Even though at 2 years group 1 had a greater loss of visual field, the decrease in peripheral visual field progressed in both groups during the 5 years of follow-up, with both 30-2 and 60-4 Humphrey visual field. The mean change in cumulative total score was -572 (635) dB versus -330 (645) dB, and the difference between groups was not statistically significant $(p=0.4)$. More than half of the eyes in group 1 were treated with ranibizumab for $\mathrm{DME}^{38}$.

An important question that this protocol answers is whether treatment with ranibizumab poses a risk of developing serious complications in the absence of PRP after 5 years of follow-up. Few eyes in both groups developed neovascular glaucoma or neovascularization of the iris, and the incidence rate was similar. The overall rate of retinal detachment (mainly tractional) was highest in group 1 (15 versus $6 \%$ ). At least half of the eyes in both groups developed some degree of vitreous hemorrhage, but only $42 \%$ in group 1 and $22 \%$ in group 2 required vitrectomy ${ }^{38}$.

In conclusion, this protocol demonstrates that ranibizumab is not inferior to PRP in terms of final BCVA, so both therapies are adequate in the treatment of PDR, mainly for those with coexisting DME. When selecting a treatment, it is important to keep in mind individual factors of the patient, such as the possibility of close monitoring, costs and metabolic control status.

\section{Protocol T}

Among the wide variety of antiangiogenic agents available, aflibercept, bevacizumab, and ranibizumab 
are the most frequently used. All three have shown an important benefit and an adequate safety profile for the treatment of DME.

In this protocol, the three agents were compared. Patients $(n=660)$ were randomized into 3 groups: 1) $2.0 \mathrm{mg}$ aflibercept, 2) $1.25 \mathrm{mg}$ bevacizumab or 3) 0.3 $\mathrm{mg}$ ranibizumab. Doses were applied at baseline and then every 4 weeks (except if the response criteria were met during the first year), and every 4 to 16 weeks in the second year. The main objective was to evaluate the change in BCVA.

During the first year, improvement in VA was +13.3 with aflibercept, 9.7 with bevacizumab and 11.2 with ranibizumab, with a statistical but not clinically significant difference. When performing the analysis by VA ranges, in the group of VA 20/32 to 20/40 there was no statistical difference $(p>0.5)$, but if the initial VA was $20 / 50$ or worse, aflibercept was better compared to the other two drugs (aflibercept versus bevacizumab [ $p<0.001$ ], aflibercept versus ranibizumab $[p=0.003]$ ) and no difference between ranibizumab and bevacizumab ${ }^{39}$.

In the 2-year analysis, the results were similar. Patients with better initial BCVA achieved similar improvement with the three drugs. In those with an initial BCVA of 20/50 or worse, aflibercept induced the highest improvement of BCVA; however, the difference was lower than in the first year and it was not statistically significant compared to ranibizumab, but it was statistically significant compared to bevacizumab. The number of injections during the second year was less than in the first year.

It can be concluded that with any of the 3 antiangiogenic agents, there is a VA improvement, and patients with a worse VA at baseline may benefit from initial treatment with aflibercept ${ }^{40}$.

\section{Protocol U}

The benefit of antiangiogenic agents has been demonstrated for the visual improvement and the decrease of CMT in eyes with DME. However, at least between 32 and $66 \%$ of the eyes treated with monthly antiangiogenics for 6 months show persistent edema associated with low vision.

This study compared treatment with ranibizumab as monotherapy versus the addition of a dexamethasone implant in patients with persistent DME (DME treated with at least 3 loading doses of an antiangiogenic agent [bevacizumab, ranibizumab or aflibercept] within the 20 previous weeks).

Subjects were randomized ( $n=116)$ in 2 groups: 1$) 0.3 \mathrm{mg}$ ranibizumab plus a $700 \mu \mathrm{g}$ dexamethasone implant or 2 ) placebo implant plus $0.3 \mathrm{mg}$ ranibizumab. Patients were evaluated every 4 weeks for 24 weeks and the need for retreatment was re-evaluated.

VA improvement was +2.7 letters in group 1 and +3 letters in group $2(95 \% \mathrm{Cl}:-3.6$ a $2.5 ; p=0.73)$. A gain of $\geq 10$ letters occurred in $22 \%$ of patients in group 1 and in $14 \%$ in group 2. CMT reduction was $-110 \mu \mathrm{m}$ in group 1 and -62 $\mu \mathrm{m}$ in group 2. In group 1, 29\% developed intraocular hypertension compared to $<1 \%$ in group 2 ( $p<0.001)$. The total number of injections during the 24 weeks was 5.6 in group 1 and 5.7 in group 2.

In conclusion, adding dexamethasone to the antiangiogenic therapy did not show an additional visual improvement, while an additional decrease of the central macular thickness was determined when compared to the group treated with monotherapy. As it was reported in previous studies, the sustained-release dexamethasone implant predisposes to an increase in intraocular pressure, so its use should be monitored closely ${ }^{41}$.

\section{Protocols currently in development}

\section{Protocol V}

The objective of the protocol is to evaluate the treatments for DME in eyes with very good vision. It will include eyes with DME by SD-OCT and a VA $\geq 20 / 25$. Patients will be randomized to receive early treatment with antiangiogenics (aflibercept and ziv-aflibercept) or early laser with delayed antiangiogenics. And the main outcome will be to evaluate the proportion of eyes with a loss of $\geq 5$ letters in 2 years.

\section{Protocol W}

This protocol consists of administering an intravitreal antiangiogenic (aflibercept) as a preventive therapy in patients with high risk of visual loss.

The primary objective is to evaluate the safety and efficacy of antiangiogenics versus observation in eyes with severe NPDR and a VA $\geq 20 / 25$ without DME and without previous treatment, as a preventive measure for the appearance of DME or PDR.

\section{Protocol AA}

It will evaluate peripheral DR lesions by ultra-widefield imaging (UWFI) and its association with the progression of DR severity. The objective is to assess if the evaluation of peripheral lesions by UWFI allows the evaluation of $\mathrm{DR}$ and to predict the progression rate over time, 
compared with 7 standard ield stereoscopic paired photos. Eyes with untreated NPDR and without macular edema will be included. Patients will undergo annual monitoring for 4 years to evaluate the relative risk of progression of two or more severity levels in groups with and without peripheral lesions using UWFI (Optos).

\section{Protocol $A B$}

This study will compare the treatment with intravitreal antiangiogenics (aflibercept) versus early vitrectomy for the treatment of vitreous hemorrhage secondary to PDR. The main objective is to compare the VA at 6 months after each of the interventions. In the antiangiogenics group, at least 2 monthly doses will be applied upon study initiation and later, vitrectomy may be performed up to 4 months later pro re nata in case of vitreous hemorrhage persistence. PRP may be applied if antiangiogenic treatment alone does not achieve disease control. In the vitrectomy group, surgery will be performed with intraoperative PRP in the first 2 weeks after randomization. Antiangiogenic injections may be applied in case of recurrent hemorrhage.

\section{Protocol AC}

It will consist in the administration of intravitreal aflibercept versus bevacizumab with delayed aflibercept for DME with central involvement. The objective is to compare the efficacy in terms of VA change. Patients in the bevacizumab group will be candidates for changing to aflibercept after 12, 16, 20 and 24 weeks if they meet the established criteria.

\section{Protocol AD (PROMINENT study)}

The objective is to evaluate whether treatment with pemfibrate $(0.2 \mathrm{mg} / 12 \mathrm{~h}$ orally) compared to placebo reduces the rate of DR progression in type 2 diabetic patients with NPDR.

\section{Protocol GEN}

The objective of this study is to create a gene bank and to collect information about clinical phenotypes for the scientific community with the rationale that in the future this can provide enough information to evaluate susceptibility or genetic resistance regarding DR. Likewise, it is intended to determine variants that may impact on key biomarkers in the development of DME and neovascularization.

\section{Conclusion}

During the last few years, the DRCR network has provided clinical results on important research questions regarding the treatment of DME and, recently, of DR.

There has been an answer to questions about treatments and technological innovations, either directly or indirectly, which otherwise would not have been answered by the industry or by other entities.

Antiangiogenic agents have been accepted globally as the first-line treatment in the management of $\mathrm{ME}$ and have shifted focal laser as adjuvant therapy in selected cases. Also, the application of grid macular laser is now in disuse. What currently is still under discussion is the management of PDR. It is well known the non-inferiority of antiangiogenics; however, their choice as first-line therapy is still controversial. In addition, steroids have shown certain benefit, although currently, they continue to be a second-line treatment for DME.

It should be noted that the conclusions provided by the network are the result of a collaboration between a group of clinical researchers who participate in academic centers, as well as in private practices, which, at least in theory, entails the ability to correctly extrapolate the results to daily clinical practice. However, we suggest that the results should be interpreted with caution always considering the individual characteristics of each patient, our environment and our main goal, which is to obtain the greatest benefit for patients using the selected therapy.

\section{Funding}

The present work was funded by the patronage of the Fundación Hospital Nuestra Señora de la Luz, Institución de Asistencia Privada. The funding organization had no role in the design or conduct of this investigation.

\section{Conflicts of interest}

No conflicts of interest are reported by any author.

\section{Ethical disclosures}

The present work has been evaluated and authorized with the number AR-Ret-0015.

Protection of human and animal subjects. The authors declare that no experiments were performed on humans or animals for this study.

Confidentiality of data. The authors declare that no patient data appear in this article. 
Right to privacy and informed consent. The authors declare that no patient data appear in this article.

\section{References}

1. Diabetic Retinopathy Clinical Research Network. Comparison of the modified Early Treatment Diabetic Retinopathy Study and mild macular grid laser photocoagulation strategies for diabetic macular edema. Arch Ophthalmol. 2007;125(4):469-8.

2. Diabetic Retinopathy Clinical Research Network. The Relationship between OCT-measured Central Retinal Thickness and Visual Acuity in Diabetic Macular Edema. Ophthalmology. 2007:114(3):525-36.

3. Diabetic Retinopathy Clinical Research Network. Comparison of Time-Domain OCT and Fundus Photographic Assessments of Retina Thickening in Eyes with Diabetic Macular Edema. Invest Ophthalmol Vis Sci. 2008:49(5):1745-52.

4. Diabetic Retinopathy Clinical Research Network. Association of the extent of diabetic macular edema as assessed by optical coherence tomography with visual acuity and retina outcome variables. Retina. 2009; 29:300-5.

5. Diabetic Retinopathy Clinical Research Network. Association of Fluorescein Angiographic Features with Visual Acuity and with Optical Coherence Tomographic and Stereoscopic Color Fundus Photographic Features of Diabetic Macular Edema in a Randomized Clinical Trial. Retina. 2010;30(10):1627-37.

6. Diabetic Retinopathy Clinical Research Network. A Randomized Trial Comparing Intravitreal Triamcinolone Acetonide and Focal/Grid Photocoagulation for Diabetic Macular Edema. Ophthalmology. 2008;115(9):1447-9

7. Diabetic Retinopathy Clinical Research Network. Three-year Follow Up of a Randomized Trial Comparing Focal/Grid Photocoagulation and Intravitreal Triamcinolone for Diabetic Macular Edema. Arch Ophthalmol. 2009;127(3):245-51

8. Diabetic Retinopathy Clinical Research Network. Diurnal Variation in Retinal Thickening Measurement by OCT in Center-involved Diabetic Macular Edema. Arch Ophthalmol. 2006;124(12):1701-7.

9. Diabetic Retinopathy Clinical Research Network. Vitrectomy Outcomes in Eyes with Diabetic Macular Edema and Vitreomacular Traction. Ophthalmology. 2010;117(6):1087-93.e3

10. Diabetic Retinopathy Clinical Research Network. Factors associated with visual acuity outcomes after vitrectomy for diabetic macular edema. Retina. 2010:30:1488-95

11. Diabetic Retinopathy Clinical Research Network. A Randomized Trial of Peribulbar Triamcinolone Acetonide with and without Focal Photocoagulation for Mild Diabetic Macular Edema: A Pilot Study. Ophthalmology. 2007;114(6):1190-6

12. Diabetic Retinopathy Clinical Research Network. An Observational Study of the Development of Diabetic Macular Edema Following Panretinal (Scatter) Photocoagulation (PRP) Given in 1 or 4 Sittings. Arch Ophthalmol. 2009;127(2):132-40.

13. Bressler NM, Edwards AR, Antoszyk AN, Beck RW, Browning DJ, Ciardella AP, et al. Retinal Thickness on Stratus Optical Coherence Tomography in People with Diabetes and Minimal or No Diabetic Retinopathy. Am J Ophthalmol. 2008;145:894-901.

14. Diabetic Retinopathy Clinical Research Network. Observational study of subclinical diabetic macular edema. Eye. 2012;26:833-40.

15. Diabetic Retinopathy Clinical Research Network. A Phase 2 Randomized Clinical Trial of Intravitreal Bevacizumab for Diabetic Macular Edema. Ophthalmology. 2007;114(10):1860-7

16. The Diabetic Retinopathy Clinical Research Network. Randomized Tria Evaluating Ranibizumab Plus Prompt or Deferred Laser or Triamcinolone Plus Prompt Laser for Diabetic Macular Edema. Ophthalmology. 2010; 117(6):1064-77.e35

17. The Diabetic Retinopathy Clinical Research Network. Expanded 2-Year Follow-up of Ranibizumab Plus Prompt or Deferred Laser or Triamcinolone Plus Prompt Laser for Diabetic Macular Edema. Ophthalmology. 2011;118(4):609-14

18. Diabetic Retinopathy Clinical Research Network. Intravitreal Ranibizumab for Diabetic Macular Edema with Prompt vs Deferred Laser Treatment: 3-year Randomized Trial Results. Ophthalmology. 2012;119(11): 2312-8.

19. Diabetic Retinopathy Clinical Research Network. Intravitreal Ranibizumab for Diabetic Macular Edema with Prompt versus Deferred Laser Treatment: 5-Year Randomized Trial Results. Ophthalmology. 2015; 122(2):375-81.

20. Diabetic Retinopathy Clinical Research Network Randomized Tria Evaluating Short-Term Effects of Intravitreal Ranibizumab or Triamcinolone Acetonide on Macular Edema Following Focal/Grid Laser for Diabe- tic Macular Edema in Eyes Also Receiving Panretinal Photocoagulation. Retina. 2011;31(6):1009-27.

21. Diabetic Retinopathy Clinical Research Network. The Course of Response to Focal/Grid Photocoagulation for Diabetic Macular Edema. Retina. 2009;29(10):1436-43.

22. Diabetic Retinopathy Clinical Research Network. Evaluation of Visual Acuity Measurements After Autorefraction vs Manual Refraction in Eyes With and Without Diabetic Macular Edema. Arch Ophthalmol. 2012; 130(4):470-9.

23. Dagogo-Jack S, Funnell MM, Davidson J. Barriers to achieving optimal glycemic control in a multi-ethnic society: a US focus. Curr Diabetes Rev. 2006;2(3):285-93

24. Davis MD, Fisher MR, Gangnon RE, Barton F, Aiello LM, Chew EY, et al. Risk factors for high-risk proliferative diabetic retinopathy and severe visual loss: ETDRS report\#18. Invest Ophthalmol Vis Sci. 1998;39:233-52.

25. Stratton IM, Kohner EM, Aldington SJ, Turner RC, Holman RR, Manley SE, et al. UKPDS 50: risk factors for incidence and progression of retinopathy in Type II diabetes over 6 years from diagnosis. Diabetologia. 2001;44(2):156-63.

26. The Diabetes Control and Complication Trial Research Group. The effect of intensive treatment of diabetes on the development and progression of long-term complication in insulin-dependent diabetes mellitus. N Engl J Med. 1993;329:977-86.

27. Diabetic Retinopathy Clinical Research Network Writing Committee. Cluster Randomized Trial Assessing the Effect on Diabetes Control of Personalized Diabetes Complication Risk Assessment during Ophthalmology Exams. JAMA Ophthalmol. 2015;133(8):888-96.

28. Diabetic Retinopathy Clincical Research Network. Randomized clinical trial evaluating intravitreal ranibizumab or saline for vitreous hemorrhage from proliferative diabetic retinopathy. JAMA Ophthalmol. 2013; 131(3):283-93.

29. Diabetic Retinopathy Clinical Research Network. Evaluation of Results 1 Year Following Short-term Use of Ranibizumab for Vitreous Hemorrhage Due to Proliferative Diabetic Retinopathy, JAMA Ophthalmol. 2014; 132(7):889-90.

30. Diabetic Retinopathy Clinical Research Network. Retinal Thickness in People with Diabetes and Minimal or No Diabetic Retinopathy: Heidelberg Spectralis Optical Coherence Tomography. Invest Ophthalmol Vis Sci. 2012;53:8154-61.

31. The Diabetic Retinopathy Clinical Research Network. Reproducibility of Spectral Domain Optical Coherence Tomography Retinal Thickness Measurements and Conversion to Equivalent Time Domain Metrics in Diabetic Macular Edema. JAMA Ophthalmol. 2014;132(9):1113-22.

32. Bressler SB, Edwards AR, Andreoli AM, Edwards PA, Glassman AR, Ja$\mathrm{ffe} \mathrm{GJ}$, et al. Reproducibility of Optovue RTVue optical coherence tomography retinal thickness measurements and conversion to equivalent Zeiss stratus metrics in diabetic macular edema. Tran Vis Sci Tech. 2015;4(1):5

33. Diabetic Retinopathy Clinical Research Network Authors/Writing Committee. Pilot Study of Individuals With Diabetic Macular Edema Undergoing Cataract Surgery. JAMA Ophthalmol. 2014;132(2):224-6.

34. Diabetic Retinopathy Clinical Research Network Authors/Writing Committee, Baker CW, Almukhtar T, et al. Macular Edema After Cataract Surgery in Eyes Without Preoperative Central-Involved Diabetic Macular Edema. JAMA Ophthalmol. 2013;131(7):870-9.

35. Friedman SM, Almukhtar TH, Baker CW, Glassman AR, Elman MJ, Bressler NM; Diabetic Retinopathy Clinical Research Network. Topical nepafenec in eyes with non-central diabetic macular edema. Retina. $2015 ; 35(5): 944-56$

36. Diabetic Retinopathy Clinical Research Network. Panretinal Photocoagulation vs Intravitreous Ranibizumab for Proliferative Diabetic Retinopathy: A Randomized Clinical Trial, JAMA Ophthalmol. 2015;314(20):2137-46.

37. Diabetic Retinopathy Clinical Research Network. Cost-effectiveness of Intravitreous Ranibizumab Compared With Panretinal Photocoagulation for Proliferative Diabetic Retinopathy Secondary Analysis From a Diabetic Retinopathy Clinical Research Network Randomized Clinical Trial. JAMA Ophthalmol 2017;135(6):576-84.

38. Diabetic Retinopathy Clinical Research Network. Five-Year Outcomes of Panretinal Photocoagulation vs Introus Ranibizumab for Proliferative Diabetic Retinopathy: A Randomized Clinical Trial. JAMA Ophthalmol. 2018;136(10):1138-48.

39. Diabetic Retinopathy Clinical Research Network; Wells JA, Glassman AR, Ayala AR, Jampol LM, Aiello LP, Antoszyk AN, et al. Aflibercept, bevaczumab, or ranibizumab for diabetic macular edema. $\mathrm{N}$ Engl $\mathrm{J}$ Med. 2015;372:1193-203

40. Diabetic Retinopathy Clinical Research Network Aflibercept. Bevacizumab, or Ranibizumab for Diabetic Macular Edema Two-Year Results from a Comparative Effectiveness Randomized Clinical Trial. Ophthalmology. 2016;123(6):1351-9.

41. Diabetic Retinopathy Clinical Research Network. Effect of Adding Dexamethasone to Continued Ranibizumab, Treatment in Patients With Persistent Diabetic Macular Edema. JAMA Ophthalmol. 2018;136(1):29-38. 